\title{
Projeto g'laucoma - resultados parciais 2000 na região de Piraquara - PR
}

\author{
Glaucoma project - partials results 2000 in the region of Piraquara - PR
}

\author{
Kenji Sakata ${ }^{1}$ \\ Luciana Scapucin ${ }^{2}$ \\ Lisandro Massanori Sakata ${ }^{3}$ \\ Ana Cristina Alvarez Carvalho ${ }^{4}$ \\ Ian Selonke $^{5}$ \\ Viviane Mayumi Sakata ${ }^{6}$ \\ Hilton Iran Ruthes ${ }^{7}$
}

${ }^{1}$ Chefe do Setor de Glaucoma do Departamento de Oftalmo-Otorrinolaringologia do Hospital das Clínicas Universidade Federal do Paraná.

${ }^{2}$ Residente do $2^{\circ}$ ano de Oftalmologia do Hospital de Clínicas - Universidade Federal do Paraná.

${ }^{3}$ Residentes do $1^{\circ}$ ano de Oftalmologia do Hospital de Clínicas - Universidade Federal do Paraná.

${ }^{4}$ Residentes do $1^{\circ}$ ano de Oftalmologia da Faculdade Evangélica de Medicina do Paraná.

${ }^{5}$ Acadêmico $6^{\circ}$ ano de Medicina Hospital de Clínicas -

Universidade Federal do Paraná.

${ }^{6}$ Acadêmica do $5^{\circ}$ ano de Medicina da Faculdade Evangélica de Medicina do Paraná

${ }^{7}$ Residente do $3^{\circ}$ ano de Oftalmologia no Hospital de Olhos - Paraná

Endereço para correspondência: R. Itupava, 1428. Alto da Rua XV - Curitiba (PR) CEP 80040-000.

E-mail: knjsakata@hotmail.com

Recebido para publicação em 14.08.2001

Aceito para publicação em 16.01.2002

Nota Editorial: Pela análise deste trabalho e por sua anuência sobre a divulgação desta nota, agradecemos ao Dr. Nelson de Mello e Oliveira.

\begin{tabular}{|c|}
\hline RESUMO \\
\hline $\begin{array}{l}\text { Objetivo: Realizar um levantamento epidemiológico do glaucoma em po- } \\
\text { pulação de uma região brasileira tendo como grupo de estudo a população } \\
\text { acima de } 40 \text { anos do município de Piraquara - PR, analisando a importância } \\
\text { dos métodos de triagem. Métodos: Este é um estudo descritivo realizado } \\
\text { durante a primeira etapa do Projeto Glaucoma do HC-UFPR no período de } \\
\text { junho de } 1999 \text { a dezembro de } 2000 \text {. Neste estudo foram triados } 1953 \text { pa- } \\
\text { cientes acima de } 40 \text { anos, os quais foram submetidos a uma anamnese } \\
\text { dirigida, avaliações das câmaras anteriores, medida da pressão intra-ocular } \\
\text { (Po) e fundoscopia. Os pacientes suspeitos de glaucoma foram encaminha- } \\
\text { dos para retornar ao ambulatório de Oftalmologia do Hospital das Clínicas } \\
\text { da Universidade Federal do Paraná - HC-UFPR para exame oftalmológico } \\
\text { completo. Resultados: Do total de pacientes triados retornaram } 184 \text { pa- } \\
\text { cientes de } 282 \text { suspeitos. Nestes retornos foram confirmados } 73 \text { casos de } \\
\text { glaucoma primário deângulo aberto(GPAA), sendo } 35 \text { (1,79\%) de glaucoma } \\
\text { crônico simples (GCS) e } 38 \text { (1,95\%) de glaucoma depressãonormal(GPN); } \\
\text { além de } 6 \text { pacientes com diagnósticos de glaucoma secundário ou glau- } \\
\text { coma de ângulo estreito. Do grupo de GCS } 22 \text { eram mulheres e } 13 \text { eram } \\
\text { homens; já no grupo GPN } 17 \text { eram mulheres e } 22 \text { eram homens. Foi também } \\
\text { demonstrada a freqüência de hipertensos e diabéticos nos dois grupos, } \\
\text { assim como outras doenças. Foram calculadas as médias dos valores da } \\
\text { Po da espessura corneana central (ECC) que demonstraram diferença } \\
\text { estatisticamente significativa entre os dois grupos. Conclusão: Nesta } \\
\text { amostra da população, as prevalências de GCS e GPN encontradas foram } \\
\text { semelhantes, o que serve como alerta para o fato de que a fundoscopia é } \\
\text { exame muito importante na triagem para detecção do glaucoma. }\end{array}$ \\
\hline
\end{tabular}

Descritores: Glaucoma/epidemiologia; Glaucoma de ângulo aberto/epidemiologia; Glaucoma/diagnóstico; Glaucoma de ângulo aberto/diagnóstico; Fundo de olho; Triagem

\section{INTRODUÇÃO}

Glaucoma é a segunda maior causa de cegueira nos EUA(1); sendo uma das maiores causas de cegueira irreversíveis do mundo. Coral-Ghanem ${ }^{(2)}$ (1984), num estudo realizado em Santa Catarina, demonstrou que o glaucoma é a primeira causa de cegueira, seguida pela catarata ${ }^{(2)}$.

Segundo Costa et al. (1998) as causas mais freqüentes de cegueira no adulto incluem: catarata, degeneração macular senil, glaucoma e retinopatia diabética. Os custos com a prevenção da cegueira induzida por estas condições são significativamente menores quando comparados aos gastos necessários à manutenção de um indivíduo cego na idade adulta ${ }^{(3)}$.

Atualmente, sabe-se que algumas doenças são consideradas como fato- 
res de risco para o surgimento de glaucoma, tais como diabetes melitus (DM) e hipertensão arterial sistêmica (HAS); contribuindo tanto para o desenvolvimento de glaucoma de pressão normal (GPN) como glaucoma crônico simples (GCS) $)^{(4)}$.

Além de estar envolvida em uma variedade de outras complicações oculares, a DM e a HAS parecem implicar no mecanismo fisiopatológico do glaucoma e da hipertensão ocular ${ }^{(5-6)}$.

Durante anos a pressão intra-ocular elevada foi considerada condição sine qua non para o diagnóstico de glaucoma. Recentemente o valor da pressão intra-ocular, como dado isolado, na patogênese do dano glaucomatoso ao nervo óptico tem sido questionado. Este fato tem especial importância no diagnóstico de casos de glaucoma de pressão normal, onde a avaliação do disco do nervo óptico e a campimetria são exames essenciais $^{(7)}$.

Apesar das alterações decorrentes da evolução do glaucoma não serem passíveis de cura, o tratamento clínico e/ou cirúrgico quando bem indicados são capazes de prevenir a cegueira; desde que seja feito o diagnóstico precoce da doença associado à conscientização da população sobre a importância de um acompanhamento oftalmológico e tratamento adequado $^{(2)}$.

Devido à existência de poucos estudos epidemiológicos na população brasileira na literatura nacional; este estudo teve como objetivo realizar um levantamento epidemiológico do glaucoma em uma população de uma região brasileira tendo como grupo de estudo a população acima de 40 anos do município de Piraquara (região metropolitana de Curitiba - PR).

\section{MÉTODOS}

Realizou-se um estudo descritivo durante a primeira etapa do Projeto Glaucoma do Hospital de Clínicas da Universidade Federal do Paraná, no período de junho de 1999 a dezembro de 2000 no município de Piraquara - PR. Segundo o censo do IBGE de 1996, o município possui uma população de 56.057 habitantes tendo 10.485 habitantes com mais de quarenta anos. Este projeto tem como objetivo a divulgação de informações sobre o glaucoma, o atendimento oftalmológico - triagem glaucoma, além de realizar a aferição da pressão arterial e aferição da glicemia por tiras reativas, da população local com idade superior a 40 anos. Os pacientes atendidos compareciam de maneira voluntária nos atendimentos de triagem após um processo de divulgação através de folders, cartazes e faixas distribuídos em pontos de grande circulação da população. Já os pacientes suspeitos eram contatados via telefone ou visita domiciliar para o atendimento de retorno. Participam do projeto médico oftalmologistas, residentes em oftalmologia e acadêmicos de medicina; sendo este um Projeto de Extensão Universitária da UFPR.

No atendimento de triagem, os pacientes eram atendidos nas unidades de saúde locais e eram submetidos a um protocolo pré-definido que constava de: anamnese com ênfase em queixas oftalmológicas e na história pregressa de HAS, DM, oftalmológicas - principalmente o glaucoma. Após a anamnese direcionada, realizou-se a dosagem da glicemia utilizando tiras reagentes tipo Dextrostix ${ }^{\circledR}$ e Glucometer Elite ${ }^{\circledR}$, efetuando-se a leitura no aparelho glucometer ${ }^{\circledR}$ e glucometer Elite ${ }^{\circledR}$. A aferição da pressão arterial foi efetuada com o paciente sentado, após 10 minutos de repouso. Quando os níveis pressóricos apresentavam-se iguais ou superiores a $140 \times 90 \mathrm{mmHg}$, repetia-se a aferição da PA após 5 minutos.

Avaliou-se a profundidade da câmara anterior através de iluminação oblíqua por exame direto externo. Seguiu-se a instilação de uma gota de colírio anestésico e uma gota de fluoresceína (marca Allergan ${ }^{\circledast}$ ). A aferição da pressão intraocular (Po) se fez com tonômetro de aplanação de Goldmann (marca Haag Streit ${ }^{\circledR}$ ) acoplado a lâmpada de fenda por residentes do $1^{\circ}$ ano em oftalmologia do HC-UFPR. Utilizou-se como valor normal da Po limítrofe-21 mmHg ${ }^{(1)}$. Realizaram-se duas medidas consecutivas e considerou-se a média entre elas. Na lâmpada de fenda a profundidade da câmara anterior era novamente avaliada. Seguiu-se a fundoscopia, após a instilação de 1 gota de colírio de tropicamida (Alcon ${ }^{\circledR}$ ), com oftalmoscópio direto (marca Heine ${ }^{\circledR}$ e/ou Welch Allyn ${ }^{\circledR}$ ) e, em casos de suspeita de retinopatia, também a oftalmoscopia indireta binocular (marca Topcon $\left.{ }^{\circledR}\right)$. Utilizaram-se como critérios de fundo de olho compatível com glaucoma: uma escavação maior que 0,5 ; a escavação vertical maior que a horizontal; a assimetria de escavação maior que 0,2 ; a presença de hemorragias no disco óptico e a presença de "notch".

Os pacientes suspeitos de apresentarem glaucoma de pressão normal ou hipertensão ocular foram encaminhados para posterior avaliação no Hospital de Clínicas. Nesta ocasião realizou-se um exame oftalmológico completo onde se repetiram as aferições da pressão arterial, tonometria e a fundoscopia, e realizou-se também exame da acuidade visual, refração, gonioscopia e paquimetria da córnea; onde se selecionaram os pacientes com Po maior ou igual a $22 \mathrm{mmHg}$ ou quadro fundoscópico compatível com glaucoma para a realização de campo visual com perímetro computadorizado Topcon ${ }^{\circledR}$ modelo SBP 2020 , estratégia 24/2. Adotaram-se os seguintes critérios para o diagnóstico de glaucoma na perimetria: escotoma de Seidel; depressão nasal superior ou inferior; degrau nasal; escotomas arqueados superiores ou inferiores completos ou incompletos.

Também foram considerados como portadores de glaucoma os pacientes suspeitos que não compareceram ao atendimento de retorno mas que apresentavam Po acima de $28 \mathrm{mmHg}$ em um dos olhos e pacientes que apresentaram fundoscopia com escavação maior que 0,8 com "notch".

Neste trabalho foram considerados como critérios diagnósticos de HAS: 1- pacientes com história pregressa e em tratamento com medicação anti-hipertensiva; 2 - pacientes que apresentaram a média de pressão arterial superior a $140 / 90 \mathrm{mmHg}$ em duas ocasiões diferentes.

Com relação ao DM foram considerados como critérios diagnósticos: 1- pacientes com história pregressa e em tratamento com medicação específica; 2 - pacientes que apresenta- 
ram exame de glicemia por tiras reativas acima de $300 \mathrm{mg} / \mathrm{dl}$ (pacientes com glicemia entre $120-300 \mathrm{mg} / \mathrm{dl}$ eram considerados suspeitos e recebiam a orientação adequada).

Para fins de análise estatística foi realizado o teste $\mathrm{t}$ de student para amostras independentes e aleatórias.

\section{RESULTADOS}

Neste estudo foram triados 1.953 pacientes acima de 40 anos, sendo 737 (37,74\%) homens e $1.216(62,36 \%)$ mulheres. A média de idade foi de 53,86 $\pm 10,57$ anos (Tabela 1). Com relação à raça: brancos - 76,92\%; mulatos - $14,43 \%$; negros 8,42\%; amarelos - 0,23\% (Tabela 2).

Do total de pacientes triados, 282 pacientes foram diagnosticados como suspeitos de apresentarem glaucoma sendo encaminhados para o atendimento de retorno no HC. Destes 282 pacientes, $184(65,25 \%)$ compareceram ao retorno (Figura 1).

Setenta e três pacientes apresentaram diagnósticos confirmados de glaucoma de ângulo aberto (GAA), sendo 35 $(47,95 \%)$ casos de glaucoma crônico simples (GCS) e 38 $(52,05 \%)$ de glaucoma de pressão normal (GPN). Além de 3 pacientes com diagnóstico de glaucoma de ângulo fechado e mais 3 pacientes de glaucomas secundários (Tabela 3 ).

Incluídos nestes pacientes com diagnóstico confirmado de glaucoma, encontram-se 12 pacientes que não compareceram ao atendimento de retorno. Apesar deste fato, 8 deles foram diagnosticados como GCS por apresentarem Po acima de $28 \mathrm{mmHg}$ e 4 foram diagnosticados como GPN devido à presença de escavação maior que 0,8 e "notch".

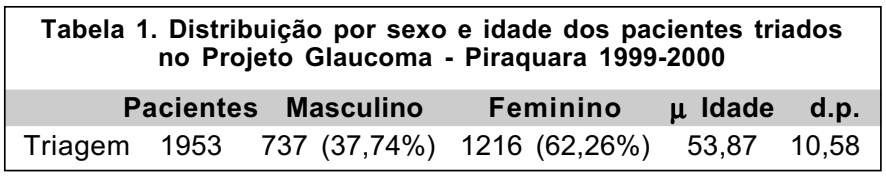

\begin{tabular}{|ccccc|}
\hline \multicolumn{5}{c|}{ Tabela 2. Raça dos pacientes triados } \\
\hline \multirow{2}{*}{ Triagem } & Brancos & Mulatos & Negros & Amarelos \\
\hline
\end{tabular}

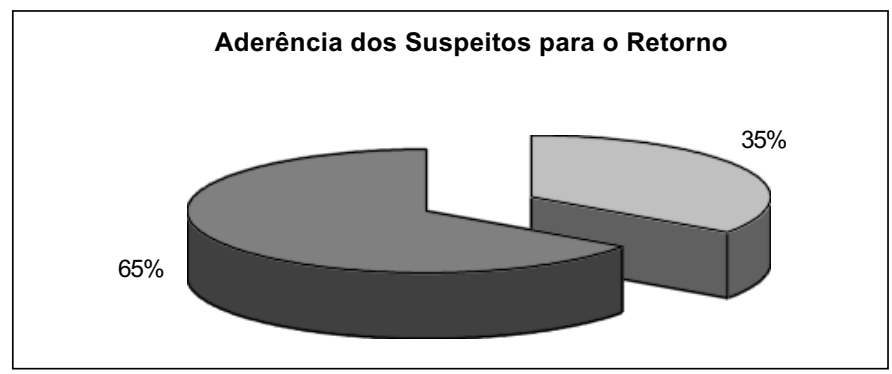

Figura 1 - $65 \%$ compareceram ao retorno e $35 \%$ não compareceram ao retorno

\begin{tabular}{|c|c|c|c|c|}
\hline & GCS & GPN & $\begin{array}{c}\text { Glauc. } \\
\text { Âng. estreito }\end{array}$ & $\begin{array}{c}\text { Glauc. } \\
\text { Secundário }\end{array}$ \\
\hline Pacientes & $\begin{array}{c}35 \\
(1,79 \%)\end{array}$ & $\begin{array}{c}38 \\
(1,95 \%)\end{array}$ & $\begin{array}{c}3 \\
(0,15 \%)\end{array}$ & $\begin{array}{c}3 \\
(0,15 \%)\end{array}$ \\
\hline
\end{tabular}

Dos 73 pacientes com glaucoma, apenas 2 deles tinham conhecimento prévio da patologia. E apenas 4 (3 - GPN) pacientes relatavam história familiar de glaucoma.

Nos 35 casos de GCS, 22 (62,86\%) eram do sexo feminino 13 $(37,14 \%)$; com uma média de idade de 56,41 (dp 11,53) anos. Com relação à raça, 69,70\% eram da raça branca; $18,18 \%$ eram mulatos; 9,09\% eram negros e 3,03\% eram amarelos.

Nos 38 casos de GPN, 17 (44,74\%) eram do sexo feminino e $21(55,26 \%)$ eram do sexo masculino; com média de idade de $54,86(\mathrm{dp} 11,80)$ anos. Com relação à raça, 86,11\% eram da raça branca; $8,33 \%$ eram mulatos; e 5,56\% eram negros (Tabela 4 ).

Dentre os casos de GCS, 23 (65,71\%) pacientes apresentavam HAS; e no grupo de GPN este número foi de $20(52,63 \%)$.

Dentre os casos de GCS existem $11(31,43 \%)$ que apresentavam DM; e no grupo de GPN este número foi de $8(21,05 \%)$ (Tabela 5).

Além da HAS, existiu um número de pacientes que apresentavam doenças cardiovasculares. Entre os GCS, 10 pacientes apresentavam doenças como: hipercolesterolemia (2); síndrome de Raynauld (2); arritmias (3); e valvopatias (3). Entre os GPN, 9 pacientes apresentavam doenças como: insuficiência cardíaca congestiva ( 3 ); hipercolesterolemia (1), síndrome de Raynauld (1); arritmias (1); infarto agudo de miocárdio (3), sendo que um deles apresentava acidente vascular cerebral associado (Tabela 5).

Dentre os 73 pacientes com GPAA confirmado, foram encontrados 5 pacientes com doenças respiratórias (DPOC). Com relação a doenças da tireóide, foi encontrado apenas um paciente (GPN) com hipertireoidismo.

Foi calculada a média da Po nos dois grupos de glaucomatosos - GCS e GPN. No primeiro grupo (GCS), a média da Po foi OD $24,19 \pm 5,86 \mathrm{mmHg}$ e no OE $23,03 \pm 4,58 \mathrm{mmHg}$. No outro

\begin{tabular}{|c|c|c|c|c|}
\hline & Masculino & Feminino & Média Idade & d.p. \\
\hline GCS & $13(37,14 \%)$ & $22(62,86 \%)$ & 56,41 & 11,54 \\
\hline GPN & $21(55,26 \%)$ & $17(44,74 \%)$ & 54,86 & 11,80 \\
\hline
\end{tabular}

\begin{tabular}{|cccc|}
\hline \multicolumn{4}{|c|}{ Tabela 5. Doenças associadas nos pacientes com GPAA do } \\
Projeto Glaucoma
\end{tabular}


grupo (GPN), a média da Po foi OD $15,05 \pm 2,86 \mathrm{mmHg}$ e no OE 15,61 $\pm 3,03 \mathrm{mmHg}$ (Tabela 6).

Também foi calculada a média da espessura corneana central (ECC) aferida no paquímetro Topcon SP-2000 P nos dois grupos. No grupo GCS, a média da ECC: OD $0,515 \pm 0,030$ e OE $0,515 \pm 0,032$. No grupo GPN, a média da ECC: OD $0,499 \pm 0,030 \mathrm{e}$ OE $0,505 \pm 0,032$. Então, após realizar o "teste t de student" para amostras independentes e aleatórias entre os dois grupos, encontraram-se as médias da ECC 0,514 e 0,499 respectivamente, e um $\mathrm{p}=0,038$ demonstrando diferença estatisticamente significativa (Tabela 7).

\section{DISCUSSÃO}

Este trabalho apresenta os primeiros resultados parciais deste Projeto Glaucoma - Piraquara 1999-2000 sendo que a população estudada representa $18,63 \%$ da população acima de 40 anos do município. Desta maneira, este trabalho mesmo utilizando as porcentagens relativas tem uma validade estatística que reflete parcialmente a realidade. Vale lembrar que, como os pacientes se apresentavam voluntariamente aos atendimentos de triagem, talvez o número de diagnósticos de glaucomatosos esteja superestimado; visto que os pacientes que apresentavam alguma forma de déficit de visão tenderiam a comparecer em maior número. Estes Bias só poderiam ser eliminados quando o numero de pacientes atendidos chegar perto dos $100 \%$.

A aderência ao atendimento de retorno dos suspeitos não foi satisfatória, apesar da conscientização dos pacientes suspeitos ser feita de maneira incisiva no final do atendimento da triagem e do transporte ao $\mathrm{HC}$ ser fornecido de maneira gratuita.

A inclusão da escavação maior ou igual a 0,5 como critério de suspeita incluiu muitos pacientes normais, o que explica o baixo número de pacientes com diagnóstico confirmados em relação aos pacientes suspeitos de glaucoma.

O nível de conhecimento com relação ao diagnóstico do glaucoma foi extremamente baixo - $2,74 \%$ dos portadores de glaucoma - refletindo o baixo número de casos já diagnosticados principalmente em comunidades mais carentes onde existe grande dificuldade para o atendimento por médicos oftalmolo-

\begin{tabular}{|lcccc|}
\hline \multicolumn{5}{|c|}{ Tabela 6. Média da PO $\begin{array}{l}(\mathrm{mmHg}) \text { dos pacientes com GPAA do } \\
\text { Projeto Glaucoma }\end{array}$} \\
Ex. & PIO OD & d.p. & PIO OE & d.p. \\
GCS & 24,19 & 5,86 & 23,03 & 4,58 \\
GPN & 15,05 & 2,86 & 15,61 & 3,03 \\
\hline
\end{tabular}

\begin{tabular}{|ccccr|}
\hline \multicolumn{5}{|c|}{ Tabela 7. Média da ECC $(\mu) \begin{array}{c}\text { nos pacientes com GPAA do projeto } \\
\text { glaucoma }\end{array}$} \\
ECC & $\boldsymbol{\mu}$ ECC OD & d.p. & $\mu$ ECC OE & d.p. \\
GCS & 0,515 & 0,030 & 0,515 & 0,032 \\
GPN & 0,499 & 0,030 & 0,505 & 0,032 \\
\hline
\end{tabular}

$\operatorname{gistas}^{(8)}$. Mesmo em pacientes em tratamento para o glaucoma o nível de conhecimento com relação a sua patologia é baixo ${ }^{(3)}$, demonstrando a necessidade de conscientização da população com relação ao glaucoma - indispensável para a aderência ao tratamento (longo, oneroso).

Da mesma maneira enquadra-se o conhecimento de história familiar pregressa do glaucoma. Relata-se que existe um componente genético na ocorrência de casos de $\mathrm{GPN}^{(9)}$, ainda que indefinido.

A prevalência do GPAA neste estudo está dentro da faixa relatada em outros trabalhos publicados na literatura. Nos estudos que envolviam uma população acima de 40 anos, como o realizado em Baltimore, onde a prevalência de GPAA encontrada foi de $1,29 \%(\mathrm{n}=5308)$. Leske et al. no estudo Barbados, a prevalência encontrada foi de $6,6 \%(\mathrm{n}=4709)$ e em outro estudo realizado no Japão $2,6 \%(\mathrm{n}=8126)^{(9)}$.

Neste estudo houve um leve predomínio de casos de GPN com relação a GCS. Apesar de todos os pacientes com diagnóstico de GPN terem apresentado duas aferições da Po em dias diferentes menor que $22 \mathrm{mmHg}$, cabe lembrar que a curva tensional diária não fez parte do protocolo do projeto. Sete pacientes apresentaram em uma das medidas PIO 20 ou $21 \mathrm{mmHg}$, ou seja perto da Po limítrofe. Então, visto a prevalência de GPN nesta população, fica evidente a importância da fundoscopia na deteç̧ão de casos suspeitos de glaucoma. Desta forma, programa de triagem do glaucoma baseado apenas na tonometria deixa muito a desejar. Na literatura a prevalência de GPN vs GCS é bastante controversa, sendo que a prevalência de GPN em relação aos casos de GAA chega a variar de 6,07\%

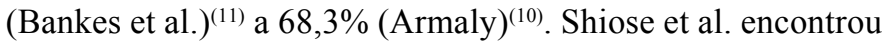
uma prevalência de $65,6 \%$ do GPN e Sommer et al. encontrou $58,8 \%{ }^{(9)}$. Enquanto que Ghanem et al. encontraram uma prevalência de $21,78 \%$ de casos de GPN em Joinvile - $\mathrm{SC}^{(2)}$.

$\mathrm{Na}$ literatura existem vários trabalhos que relatam uma maior prevalência de casos de GPN em mulheres, dado que tem como uma das possíveis justificativas o fato de que existem mais mulheres idosas do que homens ${ }^{(9)}$. Encontrou-se neste estudo um predomínio do sexo masculino nos casos de GPN $(55,26 \%)$. Isolando-se somente os homens, a porcentagem relativa dos casos de GPN em homens seria de 2,85\%, comparado com a das mulheres $1,40 \%$.

Com relação à raça, isolando-se somente os brancos, a porcentagem relativa dos casos de GCS e GPN respectivamente seria de 1,69 e $2,26 \%$. A porcentagem relativa nos mulatos seria de respectivamente 2,33 e $1,17 \%$. Nos negros 1,57 e $1,33 \%$ respectivamente. E nos amarelos 25 e $0 \%$ - provavelmente pelo $\mathrm{n}$ reduzido desta raça em nosso estudo (Tabela 8).

\begin{tabular}{lcccc|}
\multicolumn{5}{l}{ Tabela 8. Prevalência por raça dos GPAA do projeto glaucoma - } \\
porcentagem & relativa & \\
Raça & Branco & Mulatos & Negros & Amarelos \\
GCS & $1,69 \%$ & $2,33 \%$ & $1,57 \%$ & $25 \%$ \\
GPN & $2,26 \%$ & $1,17 \%$ & $1,33 \%$ & $0 \%$ \\
\hline
\end{tabular}


Com relação a HAS e doenças cardiovasculares e DM, o projeto limita-se a somente apresentar as freqüências destas patologias nos dois grupos e prefere aguardar um número maior de pacientes a fim de fornecer dados mais confiáveis, visto a grande controvérsia da relação entre estas patologias com o glaucoma.

Foi encontrada uma diferença estatisticamente significativa da ECC entre os dois grupos (GCS e GPN), como nos trabalhos de Copt et al. ${ }^{(12)}$ e Morad et al. ${ }^{(13)}$ que também demonstraram diferenças da ECC entre esses grupos; enquanto existem trabalhos como o de Wu et al. ${ }^{(14)}$ onde não foi demonstrada diferença entre os grupos. Copt et al. até re-classifica alguns casos de GPN para GCS de acordo com a Po e ECC ${ }^{(12)}$.

Este estudo é o primeiro parcial do Projeto Glaucoma e, como já citado antes, os resultados epidemiológicos mesmo que em porcentagem relativa tem seus resultados limitados ao ainda baixo número total de pacientes atendidos. Entretanto um estudo epidemiológico sobre a prevalência de glaucoma em nosso país tem sua validade visto que existem muito poucos trabalhos publicados com relação a este tema.

\section{CONCLUSÃO}

Nesta amostra da população, a prevalência de GCS de pressão alta e GPN foi de $35(1,79 \%)$ e $38(1,95 \%)$ casos respectivamente. E este resultado vem reafirmar a posição da fundoscopia como principal exame oftalmológico na detecção do glaucoma. Portanto, independente do valor da Po do paciente, a fundoscopia tem de ser realizada com muita atenção.

\section{ABSTRACT}

Purpose: To determine glaucoma epidemiology in a region of southern Brazil having as a group of study the population of Piraquara city - PR (patients over 40 years old), and analyzing the importance of the screening examination. Methods: A total of 1953 patients were screened in the Glaucoma Project (first phase) between June 1999 to December 2000. The screening process included a specific interview, examination of anterior chamber, applanation tonometry and fundoscopy. The patients screened as glaucoma suspects were submitted to a complete ophthalmologic examination at the Hospital das Clínicas UFPR. Results: Of the 1953 screened patients, 282 were classified as glaucoma suspects, but only 184 returned for the complete ophthalmologic examination. There were 73 confirmed cases of chronic open angle glaucoma; 35 (1.79\%) high- tension glaucoma cases and 38 (1.95\%) patients with normal tension glaucoma, and other 6 cases of secondary glaucoma or angle closure glaucoma. In both groups we determined gender, age and race distributions; the prevalence of diabetic and systemic hypertense population; and the IOP and CCT (central corneal thickness) averages, observing a statistically significant difference between the two groups. Conclusion: The prevalence of high-tension glaucoma and normal tension glaucoma was similar in this population sample, and this alerted us to the fact that fundoscopy is a very important examination in detecting glaucomatous patients.

Keywords: Glaucoma/epidemiology; Open angle glaucoma/ diagnosis; Glaucoma/diagnosis; Open angle glaucoma/epidemiology; Fundus oculi; Triage

\section{REFERÊNCIAS}

1. Klein BEK, Klein RÇ, Sponsel WE, Franke T, Cantor LB, Martone J, Menage MJ. Prevalence of Glaucoma. The Beaver Dam Eye Study. Ophthalmology 1992;99:1499-504.

2. Coral-Ghanem C. Levantamento de casos de Glaucoma em Joinville - Santa Catarina 1984. Arq Bras de Oftalmol 1989;52:40-3.

3. Costa VP, Almeida GV, Kara-José N. Prevenção da cegueira por glaucoma. Arq Bras Oftalmol 1998;61:356-60.

4. Leske CM, Connel MAS, Wu SY, Hyman LG, Schachat AP. Risk factors for open angle glaucoma. Arch Ophthalmol 1995;113:918-24.

5. Mitchell P, Smith W, Chey T, Stat M, Healey PR. Open-angle glaucoma and diabetes: the Blue mountains rye study, Australia. Ophthalmology 1997; 104:4:712-8.

6. Klein BEK, Klein R, Moss SE. Incidence of self reported glaucoma in people with diabetes mellitus. Br J Ophthalmol 1997;81:743-7.

7. Sommer A, Tielsch JM, Katz J, Quigley HÁ, Gottsch JD, Javitt J, Singh K. Relationship between intraocular pressure and primary open angle glaucoma among white and black Americans. The Baltimore Eye Survey. Arch Ophthalmol 1991;109:1090-5.

8. Sakata K, Vanzo LRC, Sakata LM, Bigolin S, Brick A Jr, Komatsu MC. Estudo do nível de conhecimento de pacientes glaucomatosos sobre seu diagnóstico comparado a hipertensos e diabéticos - Projeto Glaucoma. Arq Bras Oftalmol 2000;63:24.

9. Wilson MR, Martone JF, Ritch R, Shields MB, Krupin T. Epidemiology of chronic open-angle glaucoma. In: Ritch R, Shields MB, Krupin T, editors. The Glaucomas, St. Louis: Mosby; 1996. p.753-68.

10. Paterson G, Miller SJH, Paterson GD, editors Drug mechanisms in glaucoma. Boston: Little Brown; 1996.

11. Bankes JLK, Perkins ES, Tsolakis S, Wright JE. Bedford Glaucoma Survey. Br Med J 1968;1:791-6.

12. Copt RP, Thomas R, Mermoud A. Corneal thickness in ocular hypertension, primary open-angle glaucoma, and normal tension glaucoma. Arq Ophthalmol 1999;117:14-6.

13. Morad Y, Sharon E, Hefetz L, Nemet P. Corneal thickness and curvature in normal tension glaucoma. [commented on Am J Ophthalmol 1998;126:326-8]. Am J Ophthalmol 1998;125:164-8.

14. Wu LL, Suzuki Y, Ideta R, Araie M. Central corneal thickness of normal tension glaucoma patients in Japan. Japan J Ophthalmol 2000;44:643-7. 